Cite this: Phys. Chem. Chem. Phys., 2011, 13, 11620-11626

\title{
Local chemical composition of nanophase-separated polymer brushes
}

\author{
M. Filimon, ${ }^{a}$ I. Kopf, ${ }^{a}$ D. A. Schmidt,${ }^{a}$ E. Bründermann, ${ }^{a}$ J. Rühe,${ }^{b}$ S. Santer ${ }^{c}$ and \\ M. Havenith $* a$ \\ Received 2nd December 2010, Accepted 15th April 2011 \\ DOI: $10.1039 /$ c0cp02756a
}

Using scattering scanning nearfield infrared microscopy (s-SNIM), we have imaged the nanoscale phase separation of mixed polystyrene-poly(methyl methacrylate) (PS-PMMA) brushes and investigated changes in the top layer as a function of solvent exposure. We deduce that the top-layer of the mixed brushes is composed primarily of PMMA after exposure to acetone, while after exposure to toluene this changes to PS. Access to simultaneously measured topographic and chemical information allows direct correlation of the chemical morphology of the sample with topographic information. Our results demonstrate the potential of s-SNIM for chemical mapping based on distinct infrared absorption properties of polymers with a high spatial resolution of $80 \mathrm{~nm} \times 80 \mathrm{~nm}$.

\section{Introduction}

Tailoring materials with dynamic switchable responses to external fields is a challenge of modern materials science. Polymers are the perfect building blocks for manufacturing tailored surfaces. Their variable chemical functionality and the physical separation of mixed polymers into distinct phases represent a powerful and inexpensive tool for the fabrication of structured films. While physisorbed diblock copolymers can be displaced by other adsorbents, the covalent binding of polymer chains at the interface has attracted much interest. Covalently attached polymer layers, so called polymer brushes, offer enhanced stability over physisorption. ${ }^{1-6}$ Moreover, grafting two incompatible polymers randomly on a surface prevents macroscopic phase separation but still allows nano-phase segregation into nanostructures. By controlling the chain architecture, grafting density, molecular lengths of polymer brush and individual components, and interaction energy between individual polymers, well-defined patterns with characteristic sizes and shapes can form, such as spherical inclusions, wormlike or flowerlike domains. ${ }^{7-12}$ Due to the enhanced stability of polymer brushes, important properties of polymers, such as morphology, wetting, adhesion, or bio-compatibility, can be externally modified, e.g. by solvents or temperature. Such adaptive behavior is very promising for the engineering of smart surfaces for biomedical

${ }^{a}$ Department of Physical Chemistry II, Ruhr University Bochum, Universitätsstr. 150, 44780 Bochum, Germany.

E-mail: Martina.Havenith@ruhr-uni-bochum.de

${ }^{b}$ Department of Microsystems Engineering,

University of Freiburg-IMTEK, Georges-Koehler-Allee 103,

79110 Freiburg, Germany

${ }^{c}$ Department of Experimental Physics, Institute for Physics and Astronomy, University of Potsdam, Karl-Leibknecht-Str. 24/25, 14476 Potsdam, Germany applications and nanodevices. For example, a mixed brush of hydrophilic and hydrophobic homopolymers that is exposed to a hydrophilic solvent should chemically segregate, and the hydrophilic component should accumulate at the surface, which is a process called perpendicular segregation. ${ }^{13,14}$ Although reversible switching from hydrophilic to hydrophobic character has been observed, the local chemical structure has remained unknown; especially due to the switchable properties of the top-layer polymer brushes which play an important role in the wetting properties of surfaces.

In order to study the local chemical structure of polymer brush nanodomains, a chemically sensitive technique with a high lateral resolution of less than $100 \mathrm{~nm}$ is required. Fluorescence spectroscopy is highly sensitive, but it requires labeling with a chromophore which will ultimately change the structure and properties of the smart surfaces. Secondary ion mass spectroscopy can provide information about organic surfaces at the nanometre scale; however, the interpretation of spectra is difficult. Atomic force microscopy (AFM) provides information on the local topography and mechanical behavior but lacks chemical sensitivity.

Scattering scanning near-field infrared microscopy (s-SNIM) has shown to be a technique which allows chemical mapping with $\mathrm{nm}$ lateral resolution. Combining infrared spectroscopy, which has been very successfully used in spectroscopic studies of polymer materials, with the high surface sensitivity and high spatial resolution of an AFM allows us to image the chemical structure at the surface of a mixed polymer brush, including lateral and perpendicular segregation. ${ }^{15}$ s-SNIM is well-suited for non-invasively studying soft matter as it is a label-free technique with lateral resolution of $30 \mathrm{~nm} \times 30 \mathrm{~nm},{ }^{16}$ and has been used to characterize different systems ranging from materials science such as ion-implanted semiconductor surfaces $^{16-18}$ and carbon-like nanoparticles ${ }^{19,26}$ to polymer 
blends ${ }^{20-23}$ to biologically relevant systems such as SAMs, ${ }^{24,25}$ lipids $^{26}$ and a single virus. ${ }^{27}$

In this paper we demonstrate that s-SNIM offers the opportunity to record the local chemical composition of the top-layer of polymer brushes. Thereby, we can detect changes in the surface chemical structure of two different kinds of mixed PS-PMMA brushes exposed to different selective solvents. By correlating simultaneous measurements of the topography with chemical information, we can map the chemical morphology of polymer surfaces now with high lateral resolution. Visualizing the chemical morphology of a smart polymer surface is critical for understanding the structure and dynamics of polymer interfaces on the nanoscale and can be used as an analytical tool for quantitatively characterizing surface properties on the nanometre scale; thereby aiding the future design of novel materials.

\section{Material and methods}

\section{Experimental set up}

A schematic of our s-SNIM set-up is shown in Fig. 1A. A commercially available dynamic-mode atomic force microscope (AFM) (Nanotec Electronics) was modified to meet our specific applications. Gold-coated cantilevers (MikroMasch $\mathrm{NSC} 16 / \mathrm{Cr}-\mathrm{Au}$ ) with a tip curvature radius of $40 \mathrm{~nm}$ and a fundamental resonance frequency $\left(f_{0}\right)$ of about $170 \mathrm{kHz}$ are used. The cantilever is illuminated by a p-polarized beam from a home built liquid nitrogen cooled sealed-off CO-laser. ${ }^{28,29}$ The IR light is focused on the AFM tip by an off-axis parabolic mirror (focal length $101 \mathrm{~mm}$ ) onto the tip. P-polarized light ensures better coupling of the electric-field vector to the tip. The laser is line-tunable in the spectral region from 1600 to $2100 \mathrm{~cm}^{-1}(4.8-6.3 \mu \mathrm{m})$, with a typical output power of $1 \mathrm{~W}$. We attenuated the output power to $300 \mathrm{~mW}$, as measured at the laser output, for all laser lines used in our measurements. The laser provides more than 100 lines with a typical frequency separation of $3 \mathrm{~cm}^{-1}$ between adjacent laser lines. Using $\mathrm{CO}$ isotopes, we can increase the number of available laser lines to about 400. The light scattered from the tip is collected by a $\mathrm{CaF}_{2}$ lens and focused onto a liquid nitrogen cooled mercury cadmium telluride detector (MCT, Judson Technologies). Due to the oscillation of the tip, the scattered light is amplitude modulated. We use a lock-in amplifier for phase sensitive detection on the first harmonic (i.e. $2 f_{0}=340 \mathrm{kHz}$ ) of the cantilever oscillation frequency. The near-field signal increases non-linearly as a function of the sample-tip distance (see Fig. 1B), whereas the scattered light, e.g. from the shaft of the cantilever, shows a weaker non-linearity. ${ }^{30}$ If the oscillating cantilever touches the surface, it is possible to induce non-harmonic vibrations in the cantilever, leading to an increased contribution of anharmonically modulated reflected light from the cantilever's top surface. This should be avoided because it would also contribute to the expected near-field signal increase close to the surface, even when demodulated at $2 f_{0}$. Thus, the tip-sample distance is chosen such that the oscillating cantilever does not touch the sample surface, but at the same time provides the largest increase in the near-field signal. This is confirmed by monitoring the cantilever amplitude during approach to the surface and stopping the approach just before the amplitude deviates from the value far away from the sample surface; i.e. the tip-sample set point distance. Detection on the first harmonic effectively suppresses the background signal and increases the signalto-noise ratio. ${ }^{30}$ Each image was recorded at a scan rate of $0.5 \mathrm{~Hz}$ per line with an integrated time of $1 \mathrm{~ms}$ per point and pixel dimensions of $9.7 \times 9.7 \mathrm{~nm}^{2}$. Our set-up allows simultaneous recording of topography and near-field infrared scattering amplitude. All measurements were carried out in ambient conditions. Further details of our experimental set-up and data analysis can be found in ref. 19, 24-26, 31 and 32.

\section{Sample preparation}

Two types of mixed polystyrene-poly(methyl methacrylate) (PS-PMMA) brushes have been investigated: conventional and so-called Y-shaped mixed brushes, schematically shown in Fig. 2B and C, respectively. Conventional PS-PMMA mixed brushes were synthesized using surface-initiated radical polymerization ("grafting from"), described in detail in ref. 3-5, 13 and 14. Briefly, after initially modifying the silicon substrate surface with a dimethylchlorosilylpropyl 4-isobutyronitrile-4cyano pentanoate (ACMS) initiator ${ }^{33}$ and growing the PS chains, the reaction is stopped before all the initiator sites have been consumed and the remaining initiator, which is randomly distributed over the surface, is utilized for the growth of PMMA chains. In this case, each of the surface-attached
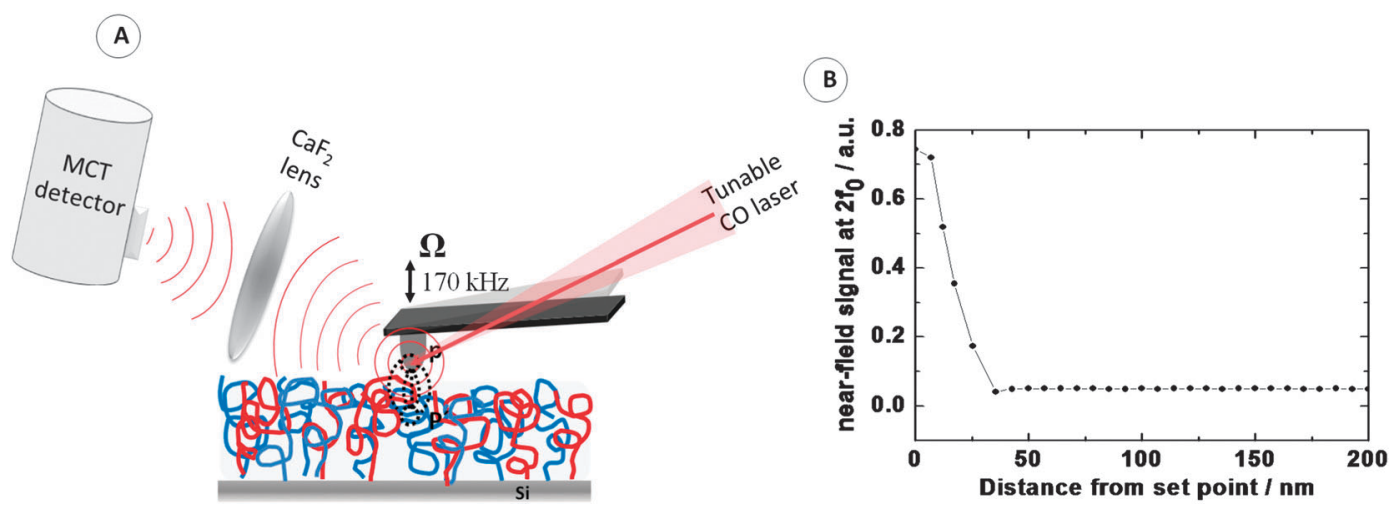

Fig. 1 (A) Schematic of the scattering scanning near-field infrared microscope. (B) Distance dependence of the scattered IR signal demodulated at twice the oscillation frequency $\left(2 f_{0}\right.$, see text for details). 


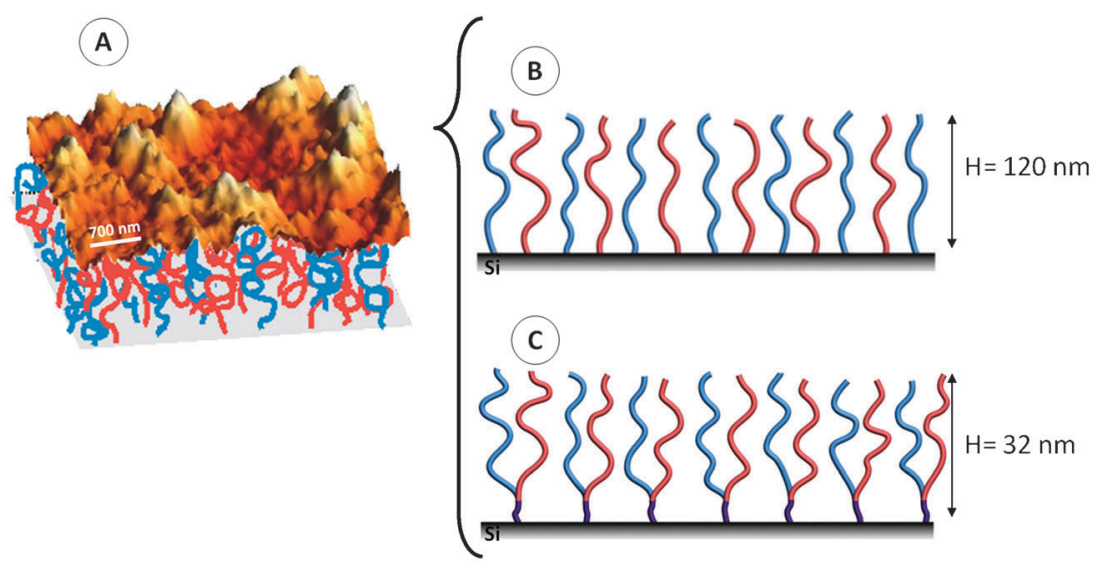

Fig. 2 (A) Schematic representation of PS-PMMA mixed brushes with 3D topography overlaid. (B) Conventional PS-PMMA mixed brushes, and (C) Y-shaped PS-PMMA brushes.

initiator groups starts the growth of either a PS or a PMMA chain in a random fashion (see schematic in Fig. 2B). The average molecular weight of the PMMA $\left(M_{n}^{\text {PMMA }}\right)$ and PS chains $\left(M_{n}{ }^{\mathrm{PS}}\right)$ is $0.9 \times 10^{6} \mathrm{~g} \mathrm{~mol}^{-1}$ and $1 \times 10^{6} \mathrm{~g} \mathrm{~mol}^{-1}$, respectively, while the total grafting density is 0.07 chains $\mathrm{nm}^{-2}$.

In Y-shaped PS-PMMA brushes, two chains of different types are attached to the same anchor group on the substrate. The synthesis starts from an asymmetric difunctional initiatorterminated self-assembled monolayer (Y-SAM) and combines two different prominent radical polymerization techniques: atom transfer radical polymerization (ATRP) and nitroxidemediated radical polymerization (NMRP). ${ }^{6}$ The Y-SAM is designed to guarantee that the two initiators for PS and PMMA are equally distributed to ensure good mixing of the homopolymers. The PMMA section of the Y brush was grown first from the Y-SAM by ATRP at $75{ }^{\circ} \mathrm{C}$ followed by the growth of PS by NMRP at $115^{\circ} \mathrm{C}^{6}$ The average molecular weight of the PMMA chains $\left(M_{n}^{\text {PMMA }}\right)$ is $2.3 \times 10^{4} \mathrm{~g} \mathrm{~mol}^{-1}$, while $M_{n}{ }^{\mathrm{PS}}$ is $2.1 \times 10^{4} \mathrm{~g} \mathrm{~mol}^{-1}$ resulting in slightly asymmetric lengths of the polymer chains. The grafting density is 1.0 chains $\mathrm{nm}^{-2}$. Details of the procedure are described in ref. 6 .

The total dry thickness of conventional and Y-shaped PS-PMMA mixed brushes is 120 and $32 \mathrm{~nm}$, respectively. ${ }^{9,14}$ Both conventional and Y-shaped samples were exposed to acetone and toluene. The microscopic morphology of both types of PS-PMMA mixed polymer brushes was visualized using AFM. The polymer films were exposed to a particular solvent for 1 minute, followed by drying with a flow of nitrogen gas. Acetone is a selective solvent for PMMA while toluene is a selective solvent for PS. ${ }^{9}$ After exposure of the mixed brushes to each of the solvents (acetone and toluene), the polymer brushes are "frozen" into characteristic morphologies due to nano-phase separation during rapid evaporation of the solvent in the stream of dry nitrogen gas.

\section{Results and discussions}

PMMA and PS are incompatible with each other resulting in films showing distinct nano-phase separation. The segregation depends strongly on the chemical structure and molecular properties of the brushes (e.g. molecular weight, hydrophobicity) as well as on the environmental conditions.

AFM micrographs of conventional and Y-shaped PSPMMA mixed brushes after exposure to different solvents are shown in Fig. 3. The topography of both types of brushes can adopt different morphologies depending on the solvent used. For Y-shaped PS-PMMA mixed brushes, the characteristic size of segregated patterns are much smaller than for much thicker conventional PS-PMMA mixed brushes. After treatment with acetone, AFM topography and phase show a characteristic nano-phase separation into "dimple"-like structures with both lateral and perpendicular segregation. The segregation of the two polymer components in height is due to PMMA preferentially occupying the surface layer of the brush. When the samples were exposed to toluene, which has an increased selectivity for PS, the components of the mixed polymer brushes create a laterally segregated "ripple" phase comprising worm-like domains and almost flat topographies. This behavior was previously observed and described by us in ref. 9 and 14 .

While AFM can detect nano-phase separation, it does not provide chemical information of the different polymers. As reported previously, s-SNIM provides a tool to image differences in chemical composition on a nm scale. ${ }^{15}$ The IR contrast is recorded simultaneously with the topography and phase, allowing direct correlation with topographic information.

In order to map the chemical distribution of the PS-PMMA mixed polymer brushes, the CO-laser was tuned through the $\mathrm{C}=\mathrm{O}$ absorption at $\sim 1740 \mathrm{~cm}^{-1}$, i.e. between $1675 \mathrm{~cm}^{-1}$ and $1835 \mathrm{~cm}^{-1}$. Simultaneously recorded topography and near-field infrared images of a $130 \mathrm{~nm}$ thick conventional PS-PMMA mixed brush at four representative frequencies of $1728 \mathrm{~cm}^{-1}, 1738 \mathrm{~cm}^{-1}, 1780 \mathrm{~cm}^{-1}$, and $1835 \mathrm{~cm}^{-1}$ are shown in Fig. 4. In all near-field images, the variation in the contrast is due to the absorption of IR light by the $\mathrm{C}=\mathrm{O}$ bond. The change from a weak image contrast on either side of $\sim 1740 \mathrm{~cm}^{-1}$ to the strongest contrast at $\sim 1740 \mathrm{~cm}^{-1}$ is a result of the laser being tuned through the absorption band of $\mathrm{C}=\mathrm{O}$ in PMMA, and can be seen in the top row of Fig. 4. The experimentally determined near-field infrared contrast values, $C$, can be calculated for each frequency according to 


\section{Conventional}
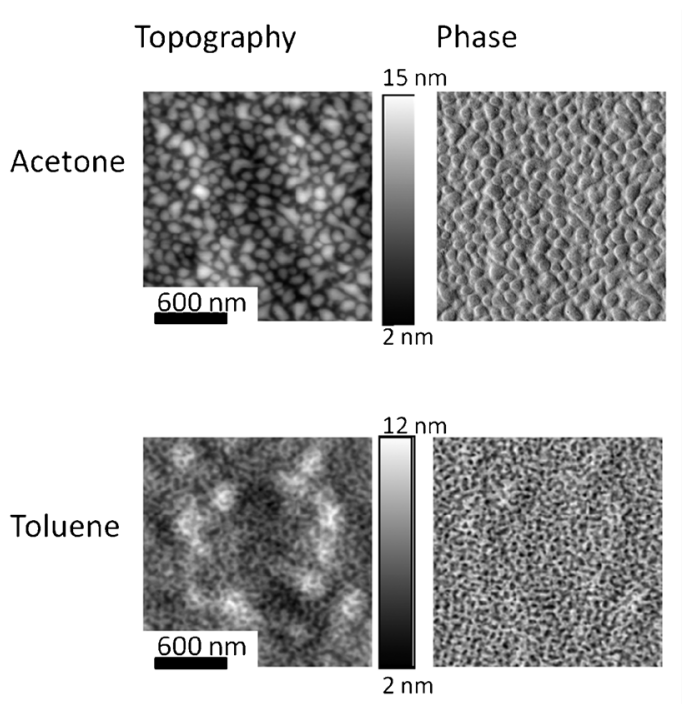

Y-Shape
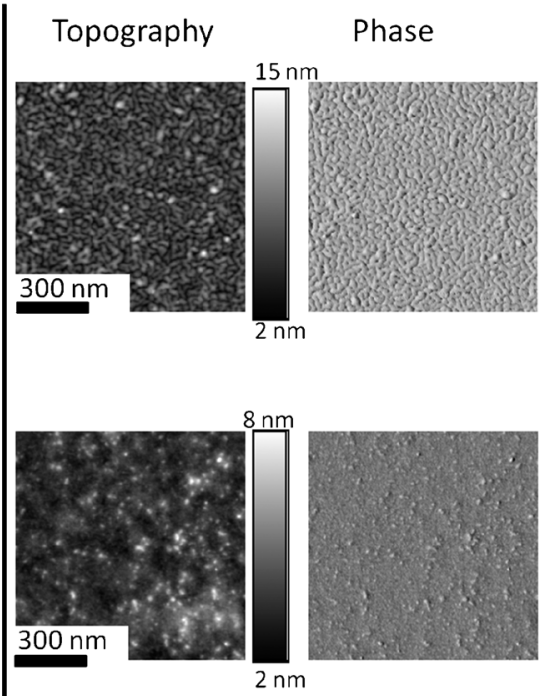

Fig. 3 AFM topography and phase micrographs of conventional (left) and Y-shaped (right) PS-PMMA mixed brushes acquired after exposure to acetone and toluene. Scan size $2 \times 2 \mu \mathrm{m}^{2}$.

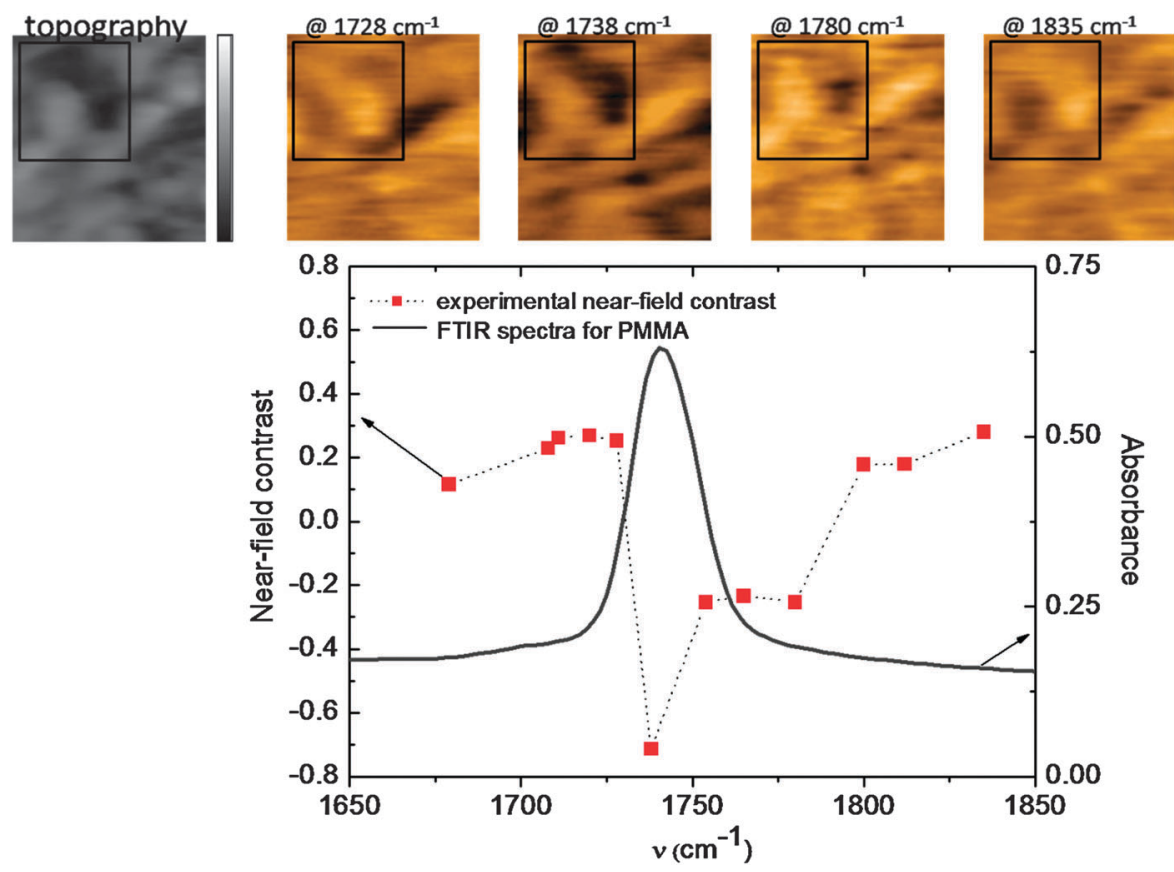

Fig. 4 Top row: AFM topography and selective IR near-field images of a $130 \mathrm{~nm}$ thick conventional PS-PMMA mixed brush; scan size: $1.5 \times 1.5 \mu \mathrm{m}^{2}$. Bottom: frequency-dependent near-field contrast of PMMA relative to PS as calculated from the near-field values within the black box. The FTIR transmission spectrum of PMMA (black curve) and the experimental near-field contrast for PMMA (red squares) is shown, while the dashed black line serves as a guide to the eye.

$C=1-\frac{I_{\text {sample }}}{I_{\text {reference }}}$, where $I_{\text {sample }}$ and $I_{\text {reference }}$ are the measured scattering intensities of PMMA and PS, respectively.

Following the same approach as in ref. 15, PS is used as a reference to calculate the spectral near-field contrast because PS has a very weak absorption band near $1750 \mathrm{~cm}^{-1}$. In contrast, PMMA shows a strong characteristic resonance at $1740 \mathrm{~cm}^{-1}$, which causes a significant change of the absorption coefficient as well as the refractive index. Both changes contribute to the observed increase in the near-field contrast at $1740 \mathrm{~cm}^{-1}$.

In general, the frequency dependence of the contrast $C$ is a convolution of absorption and dispersion in the refractive index. Fig. 4 (bottom) shows the measured frequency dependent near-field contrast (red squares) with a resonance at $1740 \mathrm{~cm}^{-1}$, which is assigned to the $\mathrm{C}=\mathrm{O}$ stretching vibration of PMMA. For comparison, the FTIR 


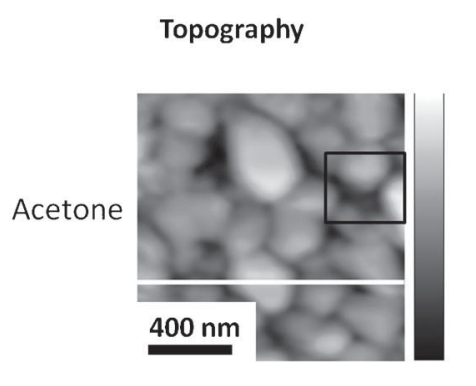

Near-field images

@ $1754 \mathrm{~cm}^{-1}$

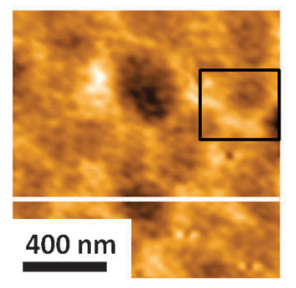

@ $1754 \mathrm{~cm}^{-1}$
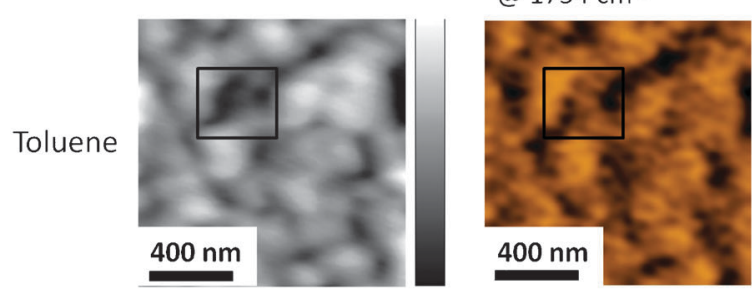

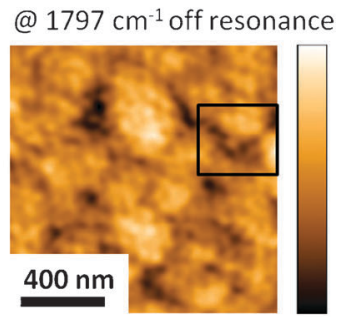

@ $1820 \mathrm{~cm}^{-1}$ off resonance

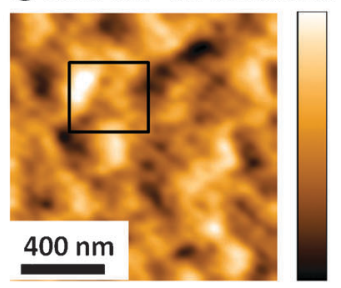

Fig. 5 Simultaneously recorded topographic and infrared near-field images on $\left(1754 \mathrm{~cm}^{-1}\right)$ and off-resonance $\left(1797 \mathrm{~cm}^{-1}\right.$ and $\left.1820 \mathrm{~cm}^{-1}\right)$ of Y-shaped PS-PMMA mixed brushes after exposure to acetone and toluene. The infrared near-field contrast of PMMA relative to PS is derived from the marked area. Scan size is $1.2 \times 1.2 \mu \mathrm{m}^{2}$. The lateral resolution has been calculated from the line profiles; $c f$. white lines (see Fig. 7).

transmission spectrum of thin-film PMMA is also shown (black curve). The frequency dependence of the recorded near-field contrast for PMMA is also in a good agreement with the theoretically predicted scattering amplitude, ${ }^{34}$ and with the results of previous studies on bulk co-block polymer films. ${ }^{20,21,23}$

Further experiments were performed with $32 \mathrm{~nm}$ thick Y-shaped PS-PMMA mixed polymer brushes after exposure to two selective solvents: acetone (selective for PMMA) and toluene (selective for PS). When using only nanomechanical information, as obtained by AFM, it is challenging to locally distinguish the two polymer components. Fig. 5 depicts the results for the measured topography and s-SNIM contrast images of acetone (top row) and toluene (bottom row) exposed Y-shaped PS-PMMA mixed polymer brushes on resonance $\left(1754 \mathrm{~cm}^{-1}\right)$ and off-resonance $\left(1797 \mathrm{~cm}^{-1}\right.$ and $\left.1820 \mathrm{~cm}^{-1}\right)$.

In both cases (acetone and toluene), the PS polymer brush near-field intensities are chosen as reference values due to the weak frequency dependence of PS, similar as in ref. 15. Using the equation for calculating the contrast, we can determine the frequency dependence of the Y-shaped PS-PMMA brushes, relative to PS, after exposure to acetone and toluene, as shown in Fig. 6. The measured contrast shows a characteristic change at the resonance frequency of the $\mathrm{C}=\mathrm{O}$ bond in PMMA. This proves that we are able to detect PMMA in the top layer, even after exposure to toluene, which is a selective solvent for PS.

We have also deduced the lateral resolution using line profiles of the topography and the IR near-field contrast at the position marked by the white lines in Fig. 5, and are shown in Fig. 7. To determine the lateral resolution for both topography and near-field contrast, we define boundaries on the rising edge of the prominent feature between 0.4 and $0.7 \mu \mathrm{m}$ (vertical lines in Fig. 7). These boundaries represent $10 \%$ above background and $90 \%$ of the maximum. The spacing between these boundaries represents the lateral resolution, which is $80 \mathrm{~nm}$ in both cases. In the case of AFM topography, the lateral resolution $(80 \mathrm{~nm})$ is limited

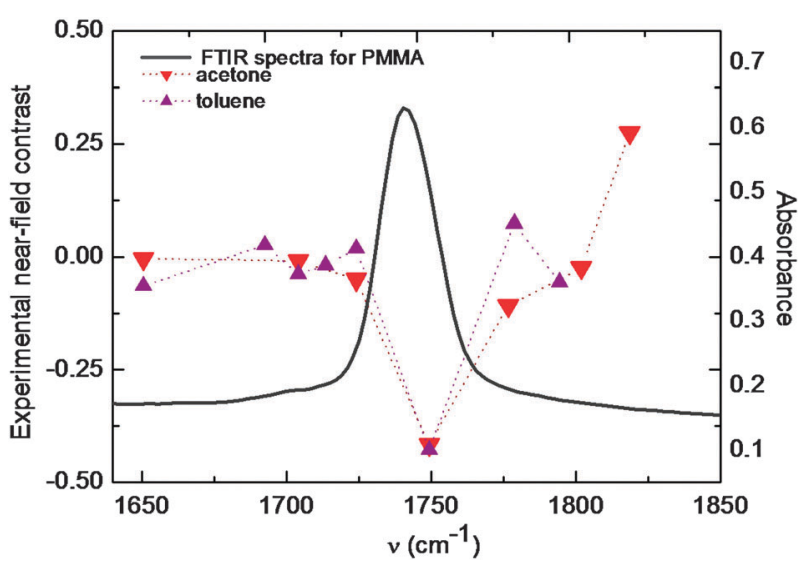

Fig. 6 Experimentally determined near-field contrast $C$ of PMMA relative to PS of $32 \mathrm{~nm}$ thick Y-shaped PS-PMMA mixed brushes after exposure to acetone (red, down triangles) and toluene (purple, up triangles) and the FTIR transmission spectrum of thin-film PMMA (black curve). Dashed lines serve as a guide to the eye.

by the probe's dimensions, while for the near-field chemical map, this corresponds to $\lambda / 72$, which is below the normal diffraction limit $(\lambda / 2)$.

By simultaneously recording both topography and nearfield scattering intensities, we can verify the chemical nature of the surface components in the Y-shaped PS-PMMA brushes after exposure to acetone (PMMA selective solvent) and toluene (PS selective solvent). Fig. 8 (bottom) shows the 3D topography (in gray scale) of the mixed PS-PMMA polymer brushes after exposure to different solvents (acetone and toluene). The corresponding near-field images at the absorption of $\mathrm{C}=\mathrm{O}\left(1754 \mathrm{~cm}^{-1}\right)$ are shown in the top row of Fig. 8 (false color images). Both polymers, PS and PMMA, can be clearly distinguished according to their distinct scattered nearfield intensity.

According to the results in Fig. 8, we deduce that the toplayer of the nanobrushes is composed primarily of PMMA 

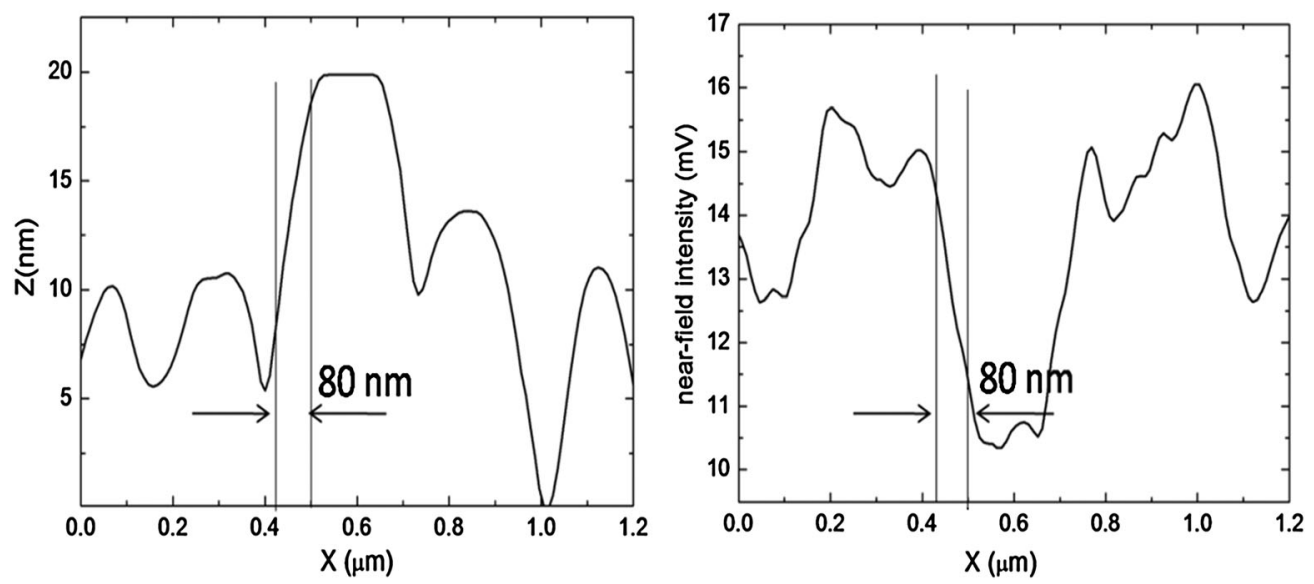

Fig. 7 Line profiles showing the topography and near-field line profile for Y-shaped PS-PMMA mixed brushes after exposure to acetone.

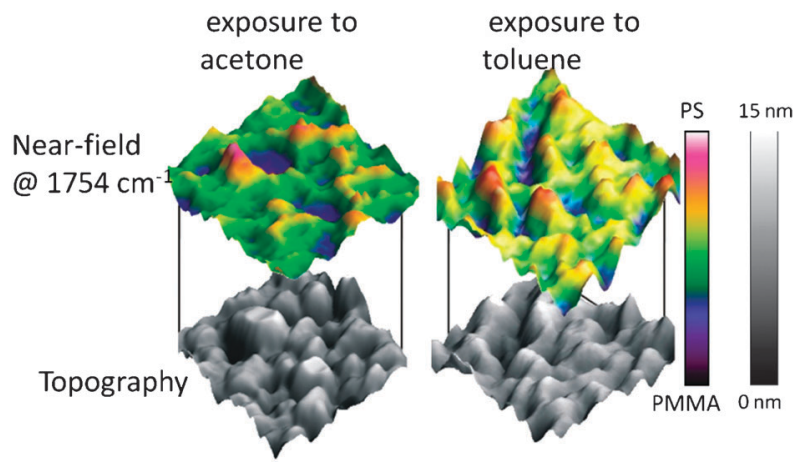

Fig. 8 3D topography (gray) and near-field (false-colored) images at $1754 \mathrm{~cm}^{-1}$ of $32 \mathrm{~nm}$ thick Y-shaped PS-PMMA brushes after exposure to acetone (left) and toluene (right). Red and yellow colors correspond to PS and blue-purple colors correspond to PMMA. The classification is based on the recorded frequency dependence of the IR near-field contrast. Scan size is $1.2 \times 1.2 \mu \mathrm{m}^{2}$.

sub-chains after exposure to acetone, while after exposure to toluene the top-layer predominantly exposes PS. Specifically we see that after exposure to acetone, the higher surface topographies (white in gray scale images) correlate well with stronger absorption (i.e. decreased scattering) in the near-field at $1754 \mathrm{~cm}^{-1}$ (blue-purple in false-color near-field images). In contrast, for the case of toluene-exposure, the higher topography features correlate well with relatively larger scattering (red-yellow in false-color images) and the stronger absorption correlates with lower topographical features. This confirms our ability to map the chemical composition of Y-shaped PS-PMMA with high lateral resolution based on their unique spectral fingerprint in the mid-IR using s-SNIM.

In summary, we demonstrated the potential of SNIM for chemical mapping of mixed polymer brush films based on characteristic IR absorption bands (here at $1754 \mathrm{~cm}^{-1}$ ) of the constituent polymers with a high spatial resolution almost 50 times better than far-field FTIR microscopy techniques. The combination of both chemical and topographic information from SNIM yields information on the local chemical composition, both laterally and vertically, of nano-phase separated polymer brushes consisting of PS and PMMA. This technique shows potential as a valuable tool for characterizing and improving the design of smart materials on the nanometre scale.

\section{Acknowledgements}

M. Filimon acknowledges financial support by Marie Curie Early Stage Research Training, in the framework of the project INTCHEM (MEST-CT-2005-020681). M. Havenith acknowledges financial support under grant $\mathrm{BMBF}$ 05KS7PC2 for innovative instrumentation of the synchrotron ANKA and Single Molecule Detection (SMD), a project funded by the EU Commission under the 7th Framework Programme (FP7-NMP-2008-SMALL-2). S. Santer thanks Prof. Bin Zhao for providing Y-shaped brushes.

\section{References}

1 (a) W. J. Brittain, J. Am. Chem. Soc., 2000, 122, 10; (b) B. Zhao and W. J. Brittain, J. Am. Chem. Soc., 1999, 121, 3557.

2 (a) S. Minko, M. Mueller, D. Usov, A. Scholl, C. Froeck and M. Stamm, Phys. Rev. Lett., 2002, 88, 035502; (b) S. Minko, M. Müller, M. Motornov, M. Nitschke, K. Grundke and M. Stamm, J. Am. Chem. Soc., 2003, 3896, 13; (c) D. Usov, V. Gruzdev, M. Nitschke, M. Stamm, O. Hoy, I. Luzinov, I. Tokarev and S. Minko, Macromolecules, 2008, 40(24), 8774.

3 S. Santer, A. Kopyshev, H.-K. Yang and J. Rühe, Macromolecules, 2006, 39(8), 3056.

4 S. Santer and J. Rühe, Polymer, 2004, 45, 8279.

5 S. Santer, A. Kopyshev, J. Donges, H.-K. Yang and J. Rühe, Langmuir, 2006, 22, 4660.

6 (a) B. Zhao and T. He, Macromolecules, 2003, 36, 8599; (b) B. Zhao, R. T. Haasch and S. MacLaren, J. Am. Chem. Soc., 2004, 126, 6124.

7 M. Motornov, R. Sheparovych, R. Lupitskyy, E. MacWilliams, O. Hoy, I. Luzinov and S. Minko, Adv. Funct. Mater., 2007, 17(14), 2307.

8 (a) L. Ionov, M. Stamm and S. Diez, Nano Lett., 2006, 6(9), 982; (b) L. Ionov and S. Diez, J. Am. Chem. Soc., 2009, 131(37), 13315.

9 A. Kopyshev, Motion of nano-particles by/on polymer brushes, $P h D$ thesis, Albert-Ludwigs Universität Freiburg, 2006.

10 R. C. Advincula, W. Brittain, K. C. Caster and J. Rühe, Polymer Brushes, Wiley-VCH, Weinheim, German, 2004.

11 B. Zhao, W. J. Brittain, W. Zhou and S. Z. D. Cheng, Macromolecules, 2000, 33, 8821.

12 D. A. V. Bout, W.-T. Yip, D. Hu, D.-K. Fu, T. M. Swager and P. F. Barbara, Science, 1997, 277(5329), 1074

13 S. Santer, A. Kopyshev, J. Donges, H.-K. Yang and J. Rühe, Adv. Mater., 2006, 18, 2359. 
14 S. Santer, A. Kopyshev, J. Donges, J. Rühe, X. Jiang, B. Zhao and M. Müller, Langmuir, 2007, 23, 279.

15 M. Filimon, I. Kopf, F. Ballout, D. A. Schmidt, E. Bründermann, J. Rühe, S. Santer and M. Havenith, Soft Matter, 2010, 6, 3764-3768.

16 B. Knoll and F. Keilmann, Nature, 1999, 399, 134.

17 B. Knoll and F. Keilmann, Appl. Phys. Lett., 2000, 77(24), 3980.

18 A. Lahrech, R. Bachelot, P. Gleyzes and A. C. Boccara, Appl. Phys. Lett., 1997, 71, 575.

19 J.-S. Samson, R. Meißner, E. Bründermann, M. Böke, J. Winter and M. Havenith, J. Appl. Phys., 2009, 105(6), 064908.

20 B. Dragnea, J. Preusser, J. M. Szarko, L. A. McDonough, S. R. Leone and W. D. Hinsberg, Appl. Surf. Sci., 2001, 175-176, 783.

21 T. Taubner, R. Hillenbrand and F. Keilmann, Appl. Phys. Lett., 2004, 85(21), 5064.

22 K. Mueller, X. Yang, M. Paulite, Z. Fakhraai, N. Gunari and G. C. Walker, Langmuir, 2008, 24, 6946.

23 M. B. Raschke, L. Molina, T. Elsaesser, D. H. Kim, W. Knoll and K. Hinrichs, ChemPhysChem, 2005, 6, 2197.

24 I. Kopf, J.-S. Samson, G. Wollny, Ch. Grunwald, E. Bründermann and H. Havenith, J. Phys. Chem. C, 2007, 111, 8166.
25 I. Kopf, C. Grunwald, E. Bründermann, L. Casali, G. Scoles and M. Havenith, J. Phys. Chem. C, 2010, 114, 1306.

26 G. Wollny, E. Bründermann, Z. Arsov, L. Quaroni and M. Havenith, Opt. Express, 2008, 16(10), 7453.

27 M. Brehm, T. Taubner, R. Hillenbrand and F. Keilamm, Nano Lett., 2006, 6(7), 1307.

28 U. Merker, P. Engels, F. Madeja, M. Havenith and W. Urban, Rev. Sci. Instrum., 1999, 70(4), 1933.

29 W. Urban, Infrared Phys. Technol., 1995, 36(1), 465.

30 B. Knoll and F. Keilmann, Opt. Commun., 2000, 182, 321.

31 E. Bründermann and M. Havenith, Annu. Rep. Prog. Chem., Sect. C, 2008, 104, 235.

32 J.-S. Samson, G. Wollny, E. Bründermann, A. Bergner, A. Hecker, G. Schwaab, A. D. Wieck and M. Havenith, Phys. Chem. Chem. Phys., 2006, 8(6), 753.

33 (a) O. Prucker and J. Rühe, Langmuir, 1998, 14(24), 6893;

(b) O. Prucker and J. Rühe, Macromolecules, 1998, 31, 592; (c) O. Prucker and J. Rühe, Macromolecules, 1998, 31, 602.

34 J. Aizpurua, T. Taubner, F. J. García de Abajo, M. Brehm and R. Hillenbrand, Opt. Express, 2008, 16(3), 1529. 